\begin{tabular}{|c|c|c|c|c|c|}
\hline $\begin{array}{l}\text { Laboratory } \\
\text { number }\end{array}$ & $\begin{array}{l}\text { Published } \\
\text { reference }\end{array}$ & $\begin{array}{l}\text { Original date or } \\
\text { other value }\end{array}$ & $\begin{array}{l}\text { Corrected date } \\
\text { or other value }\end{array}$ & $\partial \mathrm{C}^{14}, \%$ & $\begin{array}{c}\text { A.D./ } \\
\text { B.C. date }\end{array}$ \\
\hline .105 & 109:107 & $10,250 \pm 200$ & & & 8300 B.C. \\
\hline -106 & 109:107 & $10,300 \pm 200$ & & & 8350 B.C. \\
\hline . 109 & 109:106 & $3290 \pm 150$ & & & 1340 B.C. \\
\hline-110 & 109:107 & $10,180 \pm 200$ & & & 8230 B.C. \\
\hline-113 & 109:105 & $4660 \pm 150$ & & & 2710 B.C \\
\hline .115 & $109: 107$ & $9170 \pm 200$ & & & 7220 B.C. \\
\hline-122 & 109:105 & $2960 \pm 150$ & & & 1010 B.C. \\
\hline-123 & $109: 105$ & $2890 \pm 150$ & & & 940 B.C. \\
\hline-124 & 109:104 & $8050 \pm 180$ & & & 6100 B.C. \\
\hline-125 & 109:108 & $7770 \pm 180$ & & & 5820 B.C. \\
\hline-126 & $109: 104$ & $7000 \pm 180$ & & & 5050 B.C. \\
\hline-127 & 109:108 & $8700 \pm 180$ & & & 6750 B.C. \\
\hline-128 & 109:104 & $850 \pm 150$ & & & A.D. 1100 \\
\hline-129 & $109: 105$ & $4440 \pm 150$ & & & 2490 B.C. \\
\hline-130 & 109:106 & $5100 \pm 150$ & & & 3150 B.C \\
\hline-133 & $109: 105$ & $3800 \pm 150$ & & & $1850 \mathrm{~B}$ \\
\hline
\end{tabular}

${ }^{1}$ St-103A, Q-112, U-68.

${ }^{2} \mathrm{C}-159$.

${ }^{3}$ K-101, U-20, U-75, St-18.

${ }^{4} \mathrm{C}-81$.

${ }^{5}$ D-70.

\title{
University of Chicago, Institute for Nuclear Studies
}

\begin{tabular}{|c|c|c|c|c|c|}
\hline $\begin{array}{l}\text { Laboratory } \\
\text { number }\end{array}$ & $\begin{array}{l}\text { Published } \\
\text { reference }\end{array}$ & $\begin{array}{l}\text { Original date } \\
\text { or other value }\end{array}$ & $\begin{array}{c}\text { Corrected date } \\
\text { or other value }\end{array}$ & $\partial \mathrm{C}^{14}, \%$ & $\begin{array}{c}\text { A.D./ } \\
\text { B.C.date }\end{array}$ \\
\hline C-1 & a95: $77^{1}$ & $3979 \pm 350 \mathrm{~s}$ & & & 2029 B.C. \\
\hline-12 & a95:77 & $4802 \pm 210^{\mathrm{s}}$ & & & 2852 B.C. \\
\hline-36 & a95:90 & $876 \pm 250 \mathrm{~s}$ & & & A.D. 1074 \\
\hline-37 & a95:90 & $1800 \pm 500 \mathrm{~s}$ & & & A.D. 150 \\
\hline-38 & a95:90 & $5159 \pm 350 \mathrm{~s}$ & & & 3209 B.C. \\
\hline-39 & a95:90 & $8323 \pm 400^{\mathrm{s}}$ & & & 6373 B.C. \\
\hline-62 & a95:77 & $2190 \pm 450^{\mathrm{s}}$ & & & 240 B.C. \\
\hline-65 & a95:106 & $5356 \pm 350^{\mathrm{s}}$ & & & 3306 B.C. \\
\hline-72 & a95:83 & $2531 \pm 150^{\mathrm{s}}$ & & & 581 B.C. \\
\hline-75 & a95:133 & $2665 \pm 200 \mathrm{~s}$ & & & 715 B.C. \\
\hline-81 & a95:77 & $3621 \pm 180^{\mathrm{s}}$ & & & 1671 B.C. \\
\hline-101 & a95:127 & ITI + $14011 \%$ & see C-112 & & A.D. 417 \\
\hline-102 & a95:127 & & see C-112 & & A.D. 417 \\
\hline-103 & a95:134 & $1042 \pm 80^{\mathrm{s}}$ & & & A.D. 908 \\
\hline-105 & a95:105 & $>20,000$ & & & \\
\hline$-108 a$ & a95:107 & $8274 \pm 500^{\mathrm{s}}$ & & & 6324 B.C. \\
\hline-112 & a95:127 & $1533 \pm 150^{\mathrm{s}}$ & & & A.D. 417 \\
\hline-113 & a95:79 & $6707 \pm 320^{\mathrm{s}}$ & & & 4757 B.C. \\
\hline-115 & a95:79 & $3212 \pm 250^{\mathrm{s}}$ & & & 1262 B.C. \\
\hline-116 & a95:94 & $5149 \pm 300^{\mathrm{s}}$ & & & 3199 B.C. \\
\hline-119 & a95:90 & $2141 \pm 250^{\mathrm{s}}$ & & & 191 B.C. \\
\hline-120 & a95:90 & $5305 \pm 250^{\mathrm{s}}$ & & & 3355 B.C. \\
\hline-121 & a95:90 & $\mathrm{d}$ & & & \\
\hline-122 & a95:90 & $6668 \pm 250^{\mathrm{s}}$ & & & 4718 B.C. \\
\hline-126 & a95:94 & $1168 \pm 150^{\mathrm{s}}$ & & & A.D. 782 \\
\hline-136 & a95:94 & $1951 \pm 200^{\mathrm{s}}$ & & & 1 B.C. \\
\hline-137 & a95:94 & $2285 \pm 210^{\mathrm{s}}$ & & & 335 B.C. \\
\hline-139 & a95:95 & $2044 \pm 250^{\mathrm{s}}$ & & & 94 B.C. \\
\hline-143 & a95:106 & $1158 \pm 250^{\mathrm{s}}$ & & & A.D. 782 \\
\hline-150 & a95:106 & $633 \pm 150^{\mathrm{s}}$ & & & A.D. 1317 \\
\hline .151 & a95:106 & $1233 \pm 250^{\mathrm{s}}$ & & & A.D. 717 \\
\hline-152 & a95:95 & $2336 \pm 250^{\mathrm{s}}$ & & & 386 B.C. \\
\hline-153 & b95:108 & $1553 \pm 175^{\mathrm{s}}$ & & & A.D. 397 \\
\hline-154 & a95:106 & $1276 \pm 150 \mathrm{~s}$ & & & A.D. 674 \\
\hline-159 & a95:134 & $2710 \pm 130 \mathrm{~s}$ & & & 760 B.C. \\
\hline
\end{tabular}




\begin{tabular}{|c|c|c|c|c|c|}
\hline $\begin{array}{c}\text { Laboratory } \\
\text { number }\end{array}$ & $\begin{array}{l}\text { Published } \\
\text { reference }\end{array}$ & $\begin{array}{l}\text { Original date } \\
\text { or other value }\end{array}$ & $\begin{array}{l}\text { Corrected date } \\
\text { or other value }\end{array}$ & $\partial \mathrm{C}^{14}, \%$ & $\begin{array}{c}\text { A.D./ } \\
\text { B.C.date }\end{array}$ \\
\hline-164 & a95:111 & $2249 \pm 150 \mathrm{~s}$ & $(a)=C-167$ & & 299 B.C. \\
\hline-165 & a95:111 & $1752 \pm 250^{\mathrm{s}}$ & & & A.D. 198 \\
\hline-170 & $95: 111$ & $2862 \pm 250^{\mathrm{s}}$ & & & 912 B.C. \\
\hline .171 & a95:111 & $2249 \pm 250^{\mathrm{s}}$ & & & 299 B.C. \\
\hline-172 & a95:111 & $2239 \pm 250^{\mathrm{s}}$ & & & 289 B.C. \\
\hline-173 & a95:111 & $1907 \pm 250^{\mathrm{s}}$ & & & A.D. 43 \\
\hline-180 & a95:94 & $7374 \pm 500^{\mathrm{s}}$ & & & 5424 B.C. \\
\hline-183 & a95:79 & $4519 \pm 250^{\mathrm{s}}$ & & & 2569 B.C. \\
\hline-186 & a95:112 & $720 \pm 130^{\mathrm{s}}$ & & & A.D. 1230 \\
\hline-191 & a95:92 & $4930 \pm 260^{\mathrm{s}}$ & & & 2980 B.C. \\
\hline .192 & a95:92 & $2948 \pm 170^{\mathrm{s}}$ & & & 998 B.C. \\
\hline-196 & a95:128 & $3310 \pm 250^{\mathrm{s}}$ & & & 1360 B.C. \\
\hline-198 & a95:129 & $6390 \pm 300^{\mathrm{s}}$ & & & 4440 B.C. \\
\hline-199 & a95:128 & $3407 \pm 250^{\mathrm{s}}$ & & & 1457 B.C. \\
\hline-200 & a95:129 & $2422 \pm 250^{\mathrm{s}}$ & & & 472 B.C. \\
\hline-202 & a95:129 & $2565 \pm 200^{\mathrm{s}}$ & & & 615 B.C. \\
\hline-203 & a95:728 & $\mathrm{d}$ & & & \\
\hline .204 & a95:129 & $>16,000^{\mathrm{s}}$ & & & \\
\hline .205 & a95:129 & $11,003 \pm 500 \mathrm{~s}$ & & & 9053 B.C. \\
\hline-207 & a95:129 & $651 \pm 150^{\mathrm{s}}$ & & & A.D. 1299 \\
\hline-214 & a95:94 & $1509 \pm 250 \mathrm{~s}$ & & & A.D. 441 \\
\hline .216 & a95:112 & $7756 \pm 370^{\mathrm{s}}$ & & & 5806 B.C. \\
\hline-221 & a95:117 & $10,455 \pm 340^{\mathrm{s}}$ & & & 8505 B.C. \\
\hline .222 & a95:118 & $8527 \pm 250^{\mathrm{s}}$ & & & 6577 B.C. \\
\hline-247 & a95:118 & $6453 \pm 250^{\mathrm{s}}$ & & & 4503 B.C. \\
\hline-251 & a95:94 & $4900 \pm 250^{\mathrm{s}}$ & & & 2950 B.C. \\
\hline .254 & a95:94 & $5302 \pm 300 \mathrm{~s}$ & & & 3352 B.C. \\
\hline-260 & a95:127 & $973 \pm 170^{\mathrm{s}}$ & & & A.D. 977 \\
\hline-266 & a95:127 & $912 \pm 170^{\mathrm{s}}$ & & & A.D. 1038 \\
\hline-267 & a95:77 & $4883 \pm 200 \mathrm{~s}$ & & & 2933 B.C. \\
\hline-271 & a95:133 & $2257 \pm 200 \mathrm{~s}$ & & & 307 B.C. \\
\hline .276 & b95:118 & $2482 \pm 260^{\mathrm{s}}$ & & & 532 B.C. \\
\hline-277 & a95:118 & $4448 \pm 250 \mathrm{~s}$ & & & 2498 B.C. \\
\hline-278 & a95:118 & $6004 \pm 250^{\mathrm{s}}$ & & & 4054 B.C. \\
\hline-281 & a95:118 & $8660 \pm 300^{\mathrm{s}}$ & & & 6710 B.C. \\
\hline-288 & a95:92 & $4369 \pm 200^{\mathrm{s}}$ & & & 2419 B.C. \\
\hline-298 & a95:118 & $7038 \pm 350^{\mathrm{s}}$ & & & 5088 B.C. \\
\hline .299 & a95:127 & $>20,000^{\mathrm{s}}$ & & & \\
\hline-301 & b95:128 & $12,622 \pm 750^{\mathrm{s}}$ & & & 10,672 B.C. \\
\hline-302 & a95:122 & $6876 \pm 250 \mathrm{~s}$ & & & 4926 B.C. \\
\hline-308 & a95:122 & $10,877 \pm 740 \mathrm{~s}$ & & & 8927 B.C. \\
\hline-313 & a95:132 & $4257 \pm 250^{\mathrm{s}}$ & & & 2307 B.C. \\
\hline-315 & a95:132 & $3572 \pm 220 \mathrm{~s}$ & & & 1622 B.C. \\
\hline-316 & a 95:132 & $4380 \pm 270 \mathrm{~s}$ & & & 2430 B.C. \\
\hline$-318 \mathrm{a}$ & $95: 132$ & $1989 \pm 196^{\mathrm{s}}$ & $(a)=C-318$ & & 49 B.C. \\
\hline$-318 \mathrm{~b}$ & a95:132 & $3550 \pm 600^{\mathrm{s}}$ & & & 1600 B.C. \\
\hline-321 & a95:132 & $2966 \pm 300 \mathrm{~s}$ & & & 1016 B.C. \\
\hline-322 & a $95: 133$ & $3310 \pm 200^{\mathrm{s}}$ & & & 1360 B.C. \\
\hline-323 & b95:133 & $2632 \pm 300^{\mathrm{s}}$ & & & 682 B.C. \\
\hline-332 & a95:122 & $7988 \pm 420 \mathrm{~s}$ & & & 6038 B.C. \\
\hline-334 & a $95: 121$ & $7128 \pm 300 \mathrm{~s}$ & & & 5178 B.C. \\
\hline-335 & a $95: 91$ & $5962 \pm 320^{\mathrm{s}}$ & & & 4012 B.C. \\
\hline-336 & a95:105 & $9434 \pm 840^{\mathrm{s}}$ & & & 7484 B.C. \\
\hline-337 & a $95: 86$ & $11,044 \pm 500$ & & & 9094 B.C. \\
\hline-340 & a $95: 88$ & $8275 \pm 350 \mathrm{~s}$ & & & 6325 B.C. \\
\hline-341 & a95:88 & $9861 \pm 500^{\mathrm{s}}$ & & & 7911 B.C. \\
\hline-343 & a95:88 & $6044 \pm 380^{\mathrm{s}}$ & & & 4094 B.C. \\
\hline-347 & a95:87 & $3310 \pm 200^{\mathrm{s}}$ & & & 1360 B.C. \\
\hline-349 & a95:88 & $6555 \pm 280 \mathrm{~s}$ & & & 4605 B.C. \\
\hline-353 & a $95: 88$ & $9488 \pm 350^{\mathrm{s}}$ & & & 7538 B.C. \\
\hline-355 & a95:87 & $11,310 \pm 720 \mathrm{~s}$ & & & 9360 B.C. \\
\hline-356 & b95:87 & $11.787 \pm 700 \mathrm{~s}$ & & & 9837 R.C. \\
\hline-358 & a95:87 & $5824 \pm 300^{\mathrm{s}}$ & & & 3874 B.C. \\
\hline
\end{tabular}




\begin{tabular}{|c|c|c|c|c|}
\hline $\begin{array}{l}\text { Laboratory } \\
\text { number }\end{array}$ & $\begin{array}{l}\text { Published } \\
\text { reference }\end{array}$ & $\begin{array}{l}\text { Original date or } \\
\text { other value }\end{array}$ & $\begin{array}{l}\text { Corrected date } \\
\text { or other value }\end{array}$ & $\begin{array}{l}\text { A.D./ } \\
\text { B.C. date }\end{array}$ \\
\hline-362 & b95:133 & $4044 \pm 300^{\mathrm{s}}$ & & 2094 B.C. \\
\hline-363 & a95:105 & $>17,000^{\mathrm{s}}$ & & \\
\hline-364 & a95:95 & $3469 \pm 230^{\mathrm{s}}$ & & 1519 B.C. \\
\hline-365 & a95:122 & $11,437 \pm 770^{\mathrm{s}}$ & & 9487 B.C. \\
\hline-366 & a95:122 & $11,097 \pm 600 \mathrm{~s}$ & & 9147 B.C. \\
\hline-367 & a95:93 & $5383 \pm 250^{\mathrm{s}}$ & & 3433 B.C. \\
\hline-377 & a95:107 & $4283 \pm 250 \mathrm{~s}$ & & 2333 B.C. \\
\hline-378 & a $95: 134$ & $>19,000^{\mathrm{s}}$ & & \\
\hline-382 & a95:133 & $2823 \pm 500^{\mathrm{s}}$ & & 873 B.C. \\
\hline-385 & a95:106 & $12,148 \pm 700^{\mathrm{s}}$ & & $10,198 \mathrm{~B}, \mathrm{C}$ \\
\hline-406 & a95:85 & $15,516 \pm 900^{\mathrm{s}}$ & & 13,566 B.C \\
\hline .409 & a95:127 & $3018 \pm 230 \mathrm{~s}$ & & 1068 B.C. \\
\hline-417 & a95:90 & $5717 \pm 500^{\mathrm{s}}$ & & 3767 B.C. \\
\hline-418 & a95:90 & $3851 \pm 390 \mathrm{~s}$ & & 1901 B.C. \\
\hline .419 & a95:122 & $6401 \pm 230 \mathrm{~s}$ & & 4451 B.C. \\
\hline-421 & a95:129 & $4118 \pm 300 \mathrm{~s}$ & & 2168 B.C. \\
\hline-422 & a95:129 & $2244 \pm 180 \mathrm{~s}$ & & 294 B.C. \\
\hline-423 & a95:129 & $3424 \pm 230 \mathrm{~s}$ & & 1474 B.C. \\
\hline-424 & a95:130 & $2600 \pm 170^{\mathrm{s}}$ & & 650 B.C. \\
\hline-425 & a95:130 & $2223 \pm 145^{\mathrm{s}}$ & & 273 B.C. \\
\hline-426 & a95:130 & $1652 \pm 185^{\mathrm{s}}$ & & A.D. 298 \\
\hline-428 & a95:119 & $9053 \pm 350^{\mathrm{s}}$ & & 7103 B.C. \\
\hline-430 & a95:119 & $959 \pm 150 \mathrm{~s}$ & & A.D. 991 \\
\hline-432 & a $95: 86$ & $7583 \pm 380^{\mathrm{s}}$ & & 5633 B.C. \\
\hline-433 & a95:86 & $9931 \pm 350 \mathrm{~s}$ & & 7981 B.C. \\
\hline-434 & a95:87 & $8631 \pm 540 \mathrm{~s}$ & & 6681 B.C. \\
\hline-435 & b95:87 & $9425 \pm 470 \mathrm{~s}$ & & 7475 B.C. \\
\hline-438 & a95:96 & $>16,000^{\mathrm{s}}$ & & \\
\hline-440 & a95:112 & $4052 \pm 160^{\mathrm{s}}$ & & 2102 B.C. \\
\hline-444 & a95:88 & $10,851 \pm 630^{\mathrm{s}}$ & & 8901 B.C. \\
\hline-449 & b95:86 & $1129 \pm 115^{\mathrm{s}}$ & & A.D. 821 \\
\hline-450 & a95:86 & $1449 \pm 200^{\mathrm{s}}$ & & A.D. 501 \\
\hline-451 & c95:113 & $5020 \pm 300 \mathrm{~s}$ & & 3070 B.C. \\
\hline-454 & a95:126 & $7715 \pm 740^{\mathrm{s}}$ & & 5765 B.C. \\
\hline-457 & a95:77 & $6095 \pm 250^{\mathrm{s}}$ & & 4145 B.C. \\
\hline-460 & a95:133 & $1314 \pm 250^{\mathrm{s}}$ & & A.D. 636 \\
\hline-461 & a95:87 & $819 \pm 160^{s}$ & & A.D. 1131 \\
\hline-462 & a $95: 88$ & $4964 \pm 300^{\mathrm{s}}$ & & 3014 B.C. \\
\hline-463 & a95:77 & $5256 \pm 230 \mathrm{~s}$ & & 3306 B.C. \\
\hline .465 & a95:95 & $>15,000^{\mathrm{s}}$ & & \\
\hline-466 & a95:95 & $>17,000^{\mathrm{s}}$ & & \\
\hline-469 & b95:108 & $2147 \pm 150^{\mathrm{s}}$ & & 197 B.C. \\
\hline-470 & a95:107 & $10,493 \pm 1500^{\mathrm{s}}$ & & 8543 B.C. \\
\hline-471 & a $95: 107$ & $9524 \pm 450 \mathrm{~s}$ & & 7574 B.C. \\
\hline-474 & b95:105 & $10,224 \pm 510^{\mathrm{s}}$ & & 8274 B.C. \\
\hline-475 & a95:105 & $>20,000^{\mathrm{s}}$ & & \\
\hline-476 & a95:105 & $>20,000^{\mathrm{s}}$ & & \\
\hline-478 & b95:90 & $8794 \pm 550 \mathrm{~s}$ & & 6844 B.C. \\
\hline-479 & a95:88 & $>20,000^{\mathrm{s}}$ & & \\
\hline-480 & a $95: 88$ & $>17,000^{\mathrm{s}}$ & & \\
\hline .481 & a95:96 & $>17,000^{\mathrm{s}}$ & & \\
\hline-484 & a95:134 & $10.832 \pm 400^{\mathrm{s}}$ & & 8882 B.C. \\
\hline-485 & a95:134 & $8639 \pm 450^{\mathrm{s}}$ & & 6689 B.C. \\
\hline-492 & a95:83 & $8004 \pm 415^{\mathrm{s}}$ & & 6054 B.C. \\
\hline-494 & a95:84 & $8085 \pm 79,0 \mathrm{~s}$ & & 6135 B.C. \\
\hline-495 & a $95: 84$ & $8085 \pm 720 \mathrm{~s}$ & & 6135 B.C. \\
\hline-496 & a $95: 121$ & $>19.000^{\mathrm{s}}$ & & \\
\hline-497 & a95:121 & $11.283 \pm 700 \mathrm{~s}$ & & 9333 B.C. \\
\hline-500 & b95:96 & $5625 \pm 310$ & & 3675 B.C. \\
\hline-504 & a95:122 & $3656 \pm 640 \mathrm{~s}$ & & 1706 B.C. \\
\hline-505 & b95:127 & $2258 \pm 230 \mathrm{~s}$ & & 308 B.C. \\
\hline-506 & a95:127 & $1460 \pm 9 n 0^{s}$ & & A.D. 490 \\
\hline-508 & b95:96 & $>17,000$ & & \\
\hline
\end{tabular}




\begin{tabular}{|c|c|c|c|c|}
\hline $\begin{array}{l}\text { Laboratory } \\
\text { number }\end{array}$ & $\begin{array}{l}\text { Published } \\
\text { reference }\end{array}$ & $\begin{array}{l}\text { Original date or } \\
\text { other value }\end{array}$ & $\begin{array}{l}\text { Corrected date } \\
\text { or other value }\end{array}$ & $\partial \mathrm{C}^{14}, \%$ \\
\hline $\begin{array}{l}.509 \\
.510\end{array}$ & $\begin{array}{l}\mathrm{b} 95: 96 \\
\mathrm{a} 95: 96\end{array}$ & $\begin{array}{l}>19,000 \\
>20.000\end{array}$ & & \\
\hline $\begin{array}{l}-510 \\
-511\end{array}$ & $\begin{array}{l}\text { a95:96 } \\
\text { a95:113 }\end{array}$ & $\begin{array}{l}>20,000 \\
6210 \pm 450^{\mathrm{s}}\end{array}$ & & \\
\hline-515 & a95:113 & $4006 \pm 270 \mathrm{~s}$ & & \\
\hline-518 & b95:113 & $1762 \pm 420 \mathrm{~s}$ & & \\
\hline-519 & a95:113 & $2463 \pm 310 \mathrm{~s}$ & & \\
\hline-521 & a95:134 & $2211 \pm 200^{\mathrm{s}}$ & & \\
\hline-522 & a95:112 & $4052 \pm 160 \mathrm{~s}$ & & \\
\hline-523 & a95:84 & $8085 \pm 720 \mathrm{~s}$ & & \\
\hline .524 & a95:84 & $10,560 \pm 610^{\mathrm{s}}$ & & \\
\hline-525 & b95:84 & $\mathrm{d}$ & & \\
\hline-526 & b95:96 & $8513500^{\mathrm{s}}$ & & \\
\hline-528 & $95: 96$ & $16,367 \pm 1000^{\mathrm{s}}$ & & \\
\hline-535 & a95:95 & $13,842 \pm 780 \mathrm{~s}$ & & \\
\hline-536 & a95:122 & $12,168 \pm 1500^{\mathrm{s}}$ & & \\
\hline-537 & a95:122 & $11,442 \pm 640^{\mathrm{s}}$ & & \\
\hline .540 & b95:135 & $946 \pm 180^{\mathrm{s}}$ & & \\
\hline-547 & a95:83 & $8004 \pm 415^{\mathrm{s}}$ & & \\
\hline-548 & b95:135 & $4546 \pm 220^{\mathrm{s}}$ & & \\
\hline-550 & b95:77 & $6391 \pm 180^{s}$ & & \\
\hline-551 & b95:77 & $6391 \pm 180^{s}$ & & \\
\hline .554 & a95:118 & d & & \\
\hline-554 & $95: 118$ & $5737 \pm 250^{\mathrm{s}}$ & & \\
\hline-556 & a95:113 & $4508 \pm 680^{s}$ & & \\
\hline-558 & b59:107 & $9883 \pm 350^{\mathrm{s}}$ & & \\
\hline-560 & b95:127 & $5993 \pm 280^{\mathrm{s}}$ & & \\
\hline-563 & b95:127 & $2016 \pm 250^{\mathrm{s}}$ & & \\
\hline-567 & b95:112 & $1610 \pm 200 \mathrm{~s}$ & & \\
\hline-569 & b95:112 & $2816 \pm 200^{\mathrm{s}}$ & & \\
\hline-570 & b95:112 & $2048 \pm 170^{\mathrm{s}}$ & & \\
\hline-571 & b95:112 & $5605 \pm 290 \mathrm{~s}$ & & \\
\hline-572 & b95:112 & d & & \\
\hline-573 & b95:112 & $5931 \pm 310^{\mathrm{s}}$ & & \\
\hline-574 & b95:84 & $8545 \pm 500 \mathrm{~s}$ & & \\
\hline-575 & b95:95 & $>17,000^{\mathrm{s}}$ & & \\
\hline-576 & b95:84 & $1917 \pm 200 \mathrm{~s}$ & & \\
\hline-577 & b95:85 & $11,109 \pm 480^{\mathrm{s}}$ & & \\
\hline-578 & b95:86 & $15,847 \pm 1200^{\mathrm{s}}$ & & \\
\hline-579 & b95:86 & $12,986 \pm 560^{\mathrm{s}}$ & & \\
\hline-580 & b95:135 & $11,189 \pm 490 \mathrm{~s}$ & & \\
\hline-581 & b95:135 & $14,503 \pm 560 \mathrm{~s}$ & & \\
\hline-584 & b95:112 & $2223 \pm 200^{\mathrm{s}}$ & & \\
\hline-585 & b95:112 & $2145 \pm 160 \mathrm{~s}$ & & \\
\hline-587 & b95:118 & $1953 \pm 175 \mathrm{~s}$ & & \\
\hline-588 & b95:86 & $>21,000^{\mathrm{s}}$ & & \\
\hline-595 & b95:86 & $>19,000^{\mathrm{s}}$ & & \\
\hline-596 & c95:96 & $11,952 \pm 500 \mathrm{~s}$ & & \\
\hline-598 & b95:133 & $4298 \pm 230^{\mathrm{s}}$ & & \\
\hline-599 & b95:118 & $11.199 \pm 570 \mathrm{~s}$ & & \\
\hline-600 & b95:135 & $1177 \pm 175^{\mathrm{s}}$ & & \\
\hline .601 & b95:136 & $538 \pm 200 \mathrm{~s}$ & & \\
\hline-602 & b95:88 & $3798 \pm 275^{\mathrm{s}}$ & & \\
\hline-603 & b95:135 & $4513 \pm 300 \mathrm{~s}$ & & \\
\hline-604 & b95:126 & $7073 \pm 300^{\mathrm{s}}$ & & \\
\hline-606 & b95:91 & $3261 \pm 250^{\mathrm{s}}$ & & \\
\hline-607 & b95:91 & $3327 \pm 320 \mathrm{~s}$ & & \\
\hline-608 & c95:91 & $2619 \pm 220 \mathrm{~s}$ & & \\
\hline .609 & b95:119 & $11,453 \pm 600 \mathrm{~s}$ & & \\
\hline-610 & b95:119 & $11.151 \pm 570^{\mathrm{s}}$ & & \\
\hline-611 & c95:119 & $9789 \pm 630 \mathrm{~s}$ & & \\
\hline-612 & b95:112 & $2300 \pm 200 \mathrm{~s}$ & & \\
\hline .613 & c95:136 & $1361 \pm 120 \mathrm{~s}$ & & \\
\hline-615 & b95:113 & $>16,000^{\mathrm{s}}$ & & \\
\hline
\end{tabular}

A.D./

B.C. date

4260 B.C.

2056 B.C.

A.D. 188

513 B.C.

261 B.C.

2102 B.C.

6135 B.C.

8610 B.C.

6563 B.C.

14,417 B.C.

11,892 B.C.

10,218 B.C.

9492 B.C.

A.D. 1004

6054 B.C.

2596 B.C.

4441 B.C.

4441 B.C.

3787 B.C.

2558 B.C.

7933 B.C.

4043 B.C.

66 B.C.

A.D. 340

866 B.C.

98 B.C.

3655 B.C.

3981 B.C.

6595 B.C.

A.D. 33

9159 B.C.

13,897 B.C.

11,036 B.C.

9239 B.C.

12,553 B.C.

278 B.C.

195 B.C.

3 B.C.

10,002 B.C.

2348 B.C.

9249 B C

A.D. 773

A.D. 1412

1848 R.C.

2563 B.C.

5123 R.C.

1311 B C.

1377 R.C.

669 B.C.

9503 B.C.

9201 B.C.

7839 B.C.

350 B.C.

A.D. 589 


\begin{tabular}{|c|c|c|c|c|}
\hline $\begin{array}{c}\text { Laboratory } \\
\text { number }\end{array}$ & $\begin{array}{l}\text { Published } \\
\text { reference }\end{array}$ & $\begin{array}{l}\text { Original date } \\
\text { or other value }\end{array}$ & $\begin{array}{c}\text { Corrected date } \\
\text { or other value }\end{array}$ & $\begin{array}{l}\text { A.D./ } \\
\text { B.C. date }\end{array}$ \\
\hline-616 & d95:113 & $>19,000^{\mathrm{s}}$ & & \\
\hline-617 & c95:114 & $>17,800^{\mathrm{s}}$ & & \\
\hline-619 & b95:133 & $1838 \pm 190^{\mathrm{s}}$ & & A.D. 112 \\
\hline-621 & c95:89 & $2222 \pm 200^{\mathrm{s}}$ & & 272 B.C. \\
\hline-623 & c95:89 & $2562 \pm 175^{s}$ & & 612 B.C. \\
\hline-627 & c95:89 & $7965 \pm 370^{\mathrm{s}}$ & & 6015 B.C. \\
\hline-628 & b95:113 & $1879 \pm 250^{\mathrm{s}}$ & & A.D. 71 \\
\hline-629 & b95:136 & $1040 \pm 210^{\mathrm{s}}$ & & A.D. 910 \\
\hline-630 & c95:123 & $10,676 \pm 750^{\mathrm{s}}$ & & 8726 B.C. \\
\hline-631 & c95:114 & $>24,000^{\mathrm{s}}$ & & \\
\hline-632 & c95:114 & $>27,780^{\mathrm{s}}$ & & \\
\hline-635 & c95:120 & $1930 \pm 240^{\mathrm{s}}$ & & A.D. 20 \\
\hline-636 & c95:120 & $3819 \pm 160^{\mathrm{s}}$ & & 1869 B.C. \\
\hline-640 & c95:119 & $8960 \pm 340^{\mathrm{s}}$ & & 7010 B.C. \\
\hline-645 & c95:108 & $7809 \pm 400^{\mathrm{s}}$ & & 5859 B.C. \\
\hline-647 & c95:108 & $7426 \pm 600 \mathrm{~s}$ & & 5476 B.C. \\
\hline-649 & c95:109 & $4150 \pm 350^{\mathrm{s}}$ & & 2200 B.C. \\
\hline-653 & c95:97 & $12,200 \pm 500^{\mathrm{s}}$ & & 10,250 B.C. \\
\hline-657 & c95:120 & $2054 \pm 230^{\mathrm{s}}$ & & 104 B.C. \\
\hline-658 & c95:134 & $1679 \pm 200 \mathrm{~s}$ & & A.D.271 \\
\hline-659 & c95:114 & $889 \pm 100^{\mathrm{s}}$ & & A.D. 1061 \\
\hline-660 & c95:136 & $3585 \pm 260^{\mathrm{s}}$ & & 1635 B.C. \\
\hline-661 & d95:136 & $3500 \pm 225^{s}$ & & 1550 B.C. \\
\hline-662 & c95:136 & $4078 \pm 300^{s}$ & & 2128 B.C. \\
\hline-663 & c95:137 & $6310 \pm 250^{\mathrm{s}}$ & & 4360 B.C. \\
\hline-664 & c95:97 & $14,042 \pm 1000^{\mathrm{s}}$ & & 12,092 B.C. \\
\hline-667 & c95:124 & $1646 \pm 200^{\mathrm{s}}$ & & A.D. 304 \\
\hline-668 & c95:123 & $2790 \pm 350^{\mathrm{s}}$ & & 840 B.C. \\
\hline-669 & c95:137 & $3479 \pm 200^{\mathrm{s}}$ & & 1529 B.C. \\
\hline-673 & c95:115 & $1360 \pm 240^{\mathrm{s}}$ & & A.D. 590 \\
\hline-674 & c95:97 & $8200 \pm 480^{s}$ & & 6250 B.C. \\
\hline .675 & c95:98 & $1850 \pm 480^{\mathrm{s}}$ & & A.D. 100 \\
\hline-684 & c95:98 & $723 \pm 180^{\mathrm{s}}$ & & A.D. 1227 \\
\hline-687 & c95:130 & $4445 \pm 280^{\mathrm{s}}$ & & 2495 B.C. \\
\hline-688 & d95:137 & $3075 \pm 180^{\mathrm{s}}$ & & 1125 B.C. \\
\hline-689 & e95:115 & $1229 \pm 200^{\mathrm{s}}$ & & A.D. 721 \\
\hline-690 & d95:115 & $2339 \pm 150^{\mathrm{s}}$ & & 389 B.C. \\
\hline-691 & d95:115 & $2410 \pm 200^{s}$ & & 460 B.C. \\
\hline-695 & e95:115 & $1840 \pm 400^{\mathrm{s}}$ & & A.D. 110 \\
\hline-697 & c95:138 & $6098 \pm 300^{s}$ & & 4148 B.C. \\
\hline-696 & c95:128 & $333 \pm 280^{\mathrm{s}}$ & & A.D.1617 \\
\hline-698 & c95:109 & $1212 \pm 300^{\mathrm{s}}$ & & A.D. 738 \\
\hline-702 & d95:124 & $3540 \pm 220 \mathrm{~s}$ & & 1590 B.C. \\
\hline .711 & d95:124 & $3350 \pm 250^{\mathrm{s}}$ & & 1400 B.C. \\
\hline-712 & d95:124 & $3560 \pm 220^{\mathrm{s}}$ & & 1556 B.C. \\
\hline .715 & d95:125 & $3287 \pm 600^{\mathrm{s}}$ & & 1337 B.C. \\
\hline .721 & c95:138 & $1098 \pm 145^{\mathrm{s}}$ & & A.D. 582 \\
\hline-722 & $95: 125$ & $2150 \pm 400^{s}$ & & 200 B.C. \\
\hline-723 & $95: 104$ & $2377 \pm 150^{\mathrm{s}}$ & & 427 B.C. \\
\hline .728 & e95:120 & $1686 \pm 220^{\mathrm{s}}$ & & A.D. 264 \\
\hline-729 & e95:120 & $1686 \pm 220^{\mathrm{s}}$ & & A.D. 264 \\
\hline-730 & e95:120 & $1686 \pm 220^{\mathrm{s}}$ & & A.D. 264 \\
\hline-735 & e95:120 & $3172 \pm 260^{\mathrm{s}}$ & & 1762 B.C. \\
\hline-738 & c95:98 & $4289 \pm 300^{s}$ & & 2339 B.C. \\
\hline .739 & c95:99 & $4333 \pm 450^{\mathrm{s}}$ & & 2383 B.C. \\
\hline-740 & c95:99 & $4282 \pm 250^{\mathrm{s}}$ & & 2332 B.C. \\
\hline-741 & c95:99 & $3962 \pm 350^{\mathrm{s}}$ & & 2013 B.C. \\
\hline-742 & c95:79 & $6606 \pm 330^{s}$ & & 4656 B.C. \\
\hline-743 & $95: 80$ & $6695 \pm 360^{s}$ & & 4745 B.C. \\
\hline-744 & e95:80 & $5266 \pm 450^{\mathrm{s}}$ & & 3316 B.C. \\
\hline-749 & c95:89 & $5300 \pm 340^{\mathrm{s}}$ & & 3350 B.C. \\
\hline-752 & d95:81 & $3945 \pm 106^{\mathrm{s}}$ & & 1995 B.C. \\
\hline .753 & c95:78 & $5060 \pm 450^{\mathrm{s}}$ & & 3110 B.C. \\
\hline
\end{tabular}




\begin{tabular}{|c|c|c|c|c|}
\hline $\begin{array}{c}\text { Laboratory } \\
\text { number }\end{array}$ & $\begin{array}{l}\text { Published } \\
\text { reference }\end{array}$ & $\begin{array}{l}\text { Original date or } \\
\quad \text { other value }\end{array}$ & $\begin{array}{l}\text { Corrected date } \\
\text { or other value }\end{array}$ & $\partial \mathrm{C}^{14}, \%$ \\
\hline-754 & c95:78 & $5446 \pm 380^{s}$ & & \\
\hline-755 & c95:105 & $4764 \pm 250^{\mathrm{s}}$ & & \\
\hline-756 & c95:105 & $4764 \pm 250 \mathrm{~s}$ & & \\
\hline-759 & d95:98 & $2650 \pm 170 \mathrm{~s}$ & & \\
\hline-760 & d95:99 & $2169 \pm 175^{s}$ & & \\
\hline-763 & d95:116 & $6.73 \pm 0.2 \mathrm{cpm}^{\mathrm{s}}$ & & \\
\hline-764 & d95:116 & $6.39 \pm 0.5 \mathrm{cpms}^{\mathrm{s}}$ & & \\
\hline .771 & d95:116 & $6.76 \pm 0.17 \mathrm{cpms}^{\mathrm{s}}$ & & \\
\hline .777 & $\mathrm{~d} 95: 116$ & $6.83 \pm 0.12 \mathrm{cpm}^{\mathrm{s}}$ & & \\
\hline-787 & d95:116 & $6.73 \pm 0.14 \mathrm{cpm}^{\mathrm{s}}$ & & \\
\hline-790 & d95:125 & $45 \pm 150 \mathrm{~s}$ & & \\
\hline-792 & d95:128 & $3509 \pm 230^{\mathrm{s}}$ & & \\
\hline-793 & d95:128 & $4658 \pm 220^{\mathrm{s}}$ & & \\
\hline-794 & d95:93 & $4400 \pm 260^{s}$ & & \\
\hline-795 & d95:125 & $6920 \pm 500^{\mathrm{s}}$ & & \\
\hline-799 & d95:130 & $>16,000^{\mathrm{s}}$ & & \\
\hline-800 & d95:126 & $10,856 \pm 410^{\mathrm{s}}$ & & \\
\hline-801 & d95:99 & $10,972 \pm 350 \mathrm{~s}$ & & \\
\hline-809 & e95:91 & $4500 \pm 300^{\mathrm{s}}$ & & \\
\hline-810 & d95:78 & $5744 \pm 300 \mathrm{~s}$ & & \\
\hline-811 & e95:78 & $5619 \pm 280^{\mathrm{s}}$ & & \\
\hline-812 & e95:79 & $5020 \pm 290^{\mathrm{s}}$ & & \\
\hline-813 & e95:79 & $4720 \pm 310^{\mathrm{s}}$ & & \\
\hline-814 & d95:79 & $5577 \pm 300^{\mathrm{s}}$ & & \\
\hline-815 & d95:84 & $4580 \pm 200^{\mathrm{s}}$ & & \\
\hline-817 & e95:82 & $5400 \pm 325^{\mathrm{s}}$ & & \\
\hline-818 & d95:83 & $>25,000^{\mathrm{s}}$ & $>23,050$ & \\
\hline-819 & d95:85 & $5317 \pm 300^{s}$ & & \\
\hline-822 & d95:109 & $2049 \pm 180^{s}$ & & \\
\hline-823 & d95:116 & $7432 \pm 300^{\mathrm{s}}$ & & \\
\hline-824 & e95:110 & $8862 \pm 230^{\mathrm{s}}$ & & \\
\hline-827 & e95:121 & $8700 \pm 400^{s}$ & & \\
\hline-829 & e95:138 & $1854 \pm 220^{\mathrm{s}}$ & & \\
\hline-830 & e95:138 & $2139 \pm 150^{\mathrm{s}}$ & & \\
\hline-831 & e95:139 & $166 \pm 200^{\mathrm{s}}$ & & \\
\hline-832 & e95:139 & $0 \pm 250^{\mathrm{s}}$ & & \\
\hline-836 & e95:126 & $5600 \pm 600^{\mathrm{s}}$ & & \\
\hline-837 & e95:126 & $7510 \pm 600^{\mathrm{s}}$ & & \\
\hline-839 & e95:126 & $7510 \pm 600^{\mathrm{s}}$ & & \\
\hline-844 & e95:121 & $430 \pm 165^{s}$ & & \\
\hline-846 & e95:100 & $6744 \pm 530^{s}$ & & \\
\hline-848 & e95:100 & $6440 \pm 230^{\mathrm{s}}$ & & \\
\hline-849 & e95:100 & $4816 \pm 290^{\mathrm{s}}$ & & \\
\hline-850 & e95:139 & $>41,000^{\mathrm{s}}$ & & \\
\hline-851 & e95:139 & $9104 \pm 420 \mathrm{~s}$ & & \\
\hline-852 & e95:139 & $6700 \pm 5000^{s}$ & & \\
\hline-871 & e95:100 & $18,500 \pm 500^{\mathrm{s}}$ & & \\
\hline-872 & e95:100 & $>21,000^{\mathrm{s}}$ & & \\
\hline-874 & e95:100 & $1425 \pm 250^{\mathrm{s}}$ & & \\
\hline-877 & e95:87 & $3506 \pm 230 \mathrm{~s}$ & & \\
\hline-878 & e95:87 & $3713 \pm 270^{s}$ & & \\
\hline-884 & e95:131 & $3142 \pm 240^{\mathrm{s}}$ & $(e)=C .884$ & \\
\hline-886 & e95:131 & $2970 \pm 200 \mathrm{~s}$ & & \\
\hline-887 & e95:131 & $2490 \pm 300^{s}$ & & \\
\hline-891 & e95:131 & $2993 \pm 360^{s}$ & & \\
\hline-893 & e95:100 & $16,100 \pm 850 \mathrm{~s}$ & & \\
\hline .894 & e95:116 & $10,494 \pm 560^{\mathrm{s}}$ & & \\
\hline-895 & e95:117 & $15,089 \pm 1000^{\mathrm{s}}$ & & \\
\hline-896 & e95:117 & $18,000 \pm 730^{\mathrm{s}}$ & & \\
\hline-897 & e95:117 & $23,923 \pm 1800^{\mathrm{s}}$ & & \\
\hline-893 & e95:117 & $>17,800$ & & \\
\hline 899 & e95:103 & $5955^{\prime} \pm 235^{\mathrm{s}}$ & & \\
\hline .900 & e95:103 & $5268 \pm 230^{\mathrm{s}}$ & & \\
\hline
\end{tabular}

A.D./

B.C. date 3496 B.C. 2814 B.C.

2814 B.C. 700 B.C. 219 B.C.

A.D. 1905

1559 B.C.

2708 B.C.

2250 B.C.

4970 B.C.

8906 B.C.

9022 B.C.

2550 B.C.

3794 B.C.

3669 B.C.

3070 B.C.

2770 B.C.

3627 B.C.

2630 B.C.

3450 B.C.

3367 B.C.

99 B.C.

5842 B.C

6912 B.C.

6750 B.C.

A.D. 96

189 B.C.

A.D. 1784

A.D. 1950

3650 B.C.

5560 B.C.

5560 B.C.

A.D. 1520

4794 B.C.

4490 B.C.

2866 B.C.

7154 B.C.

4750 B.C.

16,550 B.C.

A.D. 525

1556 B.C.

1763 R.C.

1192 B.C.

1020 B.C.

540 B.C.

1043 B.C.

14.150 B.C.

8544 B.C.

13.139 B.C.

16,050 R.C.

21,973 B.C.

4005 P. C.

3318 B.C. 


\begin{tabular}{|c|c|c|c|c|c|}
\hline $\begin{array}{l}\text { Laboratory } \\
\text { number }\end{array}$ & $\begin{array}{l}\text { Published } \\
\text { reference }\end{array}$ & $\begin{array}{l}\text { Original date or } \\
\text { other value }\end{array}$ & $\begin{array}{l}\text { Corrected date } \\
\text { or other value }\end{array}$ & $\partial \mathrm{C}^{14}, \%$ & $\begin{array}{c}\text { A.D./ } \\
\text { B.C. date }\end{array}$ \\
\hline-903 & e95:103 & $8546 \pm 380^{\mathrm{s}}$ & & & 6596 B.C. \\
\hline-904 & e95:103 & $10,947 \pm 900^{\mathrm{s}}$ & & & 8997 B.C. \\
\hline $.904^{1}$ & e95:103 & $7800 \pm 900 \mathrm{~s}$ & & & 5850 B.C. \\
\hline-905 & e95:103 & $11,200 \pm 800^{\mathrm{s}}$ & & & 9250 B.C. \\
\hline-907 & e95:103 & $10,651 \pm 650^{\mathrm{s}}$ & & & 8701 B.C. \\
\hline .908 & e95:103 & $9101 \pm 440^{\mathrm{s}}$ & & & 7151 B.C. \\
\hline-911 & e95:140 & $3368 \pm 200^{\mathrm{s}}$ & & & 1418 B.C. \\
\hline-912 & e95:103 & $12,120 \pm 530^{\mathrm{s}}$ & & & 10,170 B.C. \\
\hline-913 & e95:103 & $13,300 \pm 900 \mathrm{~s}$ & & & 11,350 B.C. \\
\hline-914 & e95:121 & $>23,800^{\mathrm{s}}$ & & & \\
\hline-917 & e95:140 & $1506 \pm 305^{\mathrm{s}}$ & & & A.D. 444 \\
\hline-919 & e95:84 & $7420 \pm 520^{\mathrm{s}}$ & & & 5470 B.C. \\
\hline-923 & e95:104 & $2377 \pm 150^{\mathrm{s}}$ & & & 427 B.C. \\
\hline-924 & e95:140 & $11,600 \pm 700 \mathrm{~s}$ & & & 9650 B.C. \\
\hline-925 & e95:140 & $15,100 \pm 730^{\mathrm{s}}$ & & & $13,150 \mathrm{BC}$ \\
\hline-926 & e95:140 & $16,811 \pm 960^{\mathrm{s}}$ & & & 14,861 B.C. \\
\hline-927 & e95:140 & $11,700 \pm 610^{\mathrm{s}}$ & & & 9750 B.C. \\
\hline .928 & e95:104 & $1343 \pm 240^{\mathrm{s}}$ & & & A.D. 607 \\
\hline-930 & e95:110 & $2675 \pm 280 \mathrm{~s}$ & & & 725 B.C. \\
\hline-931 & e95:110 & $3100 \pm 410^{\mathrm{s}}$ & & & 1150 B.C. \\
\hline .933 & e95:111 & $2104 \pm 140^{\mathrm{s}}$ & & & 154 B.C. \\
\hline .935 & e95:104 & $>21,600$ & & & \\
\hline-937 & e95:105 & $>24,000$ & & & $>22,050$ B.C. \\
\hline-942 & e95:104 & $2780 \pm 410^{\mathrm{s}}$ & & & 830 B.C. \\
\hline-943 & e95:93 & $2830 \pm 220^{\mathrm{s}}$ & & & 880 B.C. \\
\hline-948 & e95:131 & $1485 \pm 120^{\mathrm{s}}$ & & & A.D. 465 \\
\hline-949 & e95:131 & $1521 \pm 170^{\mathrm{s}}$ & & & A.D. 429 \\
\hline
\end{tabular}

${ }^{1}$ The place (but not the page) of original publication is indicated by prefixed letters in this column: a, Science, v.113, p. 111-120, 1951; b, ibid., v. 114, p. 291-296, 1951; c, ibid., v. 116, p. 673-681, 1952; d, ibid., v. 119, p. 135-140, 1954; e, ibid., v. 120, p. 733-742, 1954.

\section{Trinity College, Dublin ${ }^{1}$}

\begin{tabular}{|c|c|c|c|c|c|}
\hline $\begin{array}{c}\text { Laboratory } \\
\text { number }\end{array}$ & $\begin{array}{l}\text { Published } \\
\text { reference }\end{array}$ & $\begin{array}{l}\text { Original date or } \\
\text { other value }\end{array}$ & $\begin{array}{l}\text { Corrected date } \\
\text { or other value }\end{array}$ & $\partial \mathrm{C}^{14}, \%$ & $\begin{array}{l}\text { A.D./ } \\
\text { B.C. date }\end{array}$ \\
\hline D-1 & $77: 27$ & $6460 \pm 200$ & & & 4510 B.C. \\
\hline-2 & $77: 27$ & $6400 \pm 200$ & & & 4450 B.C. \\
\hline-3 & $77: 28$ & $3570 \pm 170$ & & & 1620 B.C. \\
\hline-4 & $77: 27$ & $5170 \pm 190$ & & & 3220 B.C. \\
\hline-5 & $77: 28$ & $4430 \pm 140$ & & & 2180 B.C. \\
\hline-6 & $77: 28$ & $2625 \pm 130$ & & & 675 B.C. \\
\hline-7 & $77: 28$ & $2220 \pm 130$ & & & 270 B.C. \\
\hline-8 & $77: 28$ & $1725 \pm 130$ & & & A.D. 225 \\
\hline-9 & $77: 28$ & $1380 \pm 120$ & & & A.D. 570 \\
\hline-10 & $77: 28$ & $1450 \pm 130$ & & & A.D. 500 \\
\hline-11 & $77: 28$ & $500 \pm 130$ & & & A.D. 1450 \\
\hline-12 & $77: 28$ & $5160 \pm 190$ & & & 3210 B.C. \\
\hline-13 & $77: 29$ & $4970 \pm 190$ & & & 3020 B.C. \\
\hline-14 & $77: 29$ & $4550 \pm 180$ & & & 2600 B.C. \\
\hline-15 & $77: 29$ & $3665 \pm 130$ & & & 1715 B.C. \\
\hline-16 & $77: 29$ & $3720 \pm 120$ & & & 1170 B.C. \\
\hline-17 & $77: 29$ & $1220 \pm 120$ & & & A.D. 730 \\
\hline-18 & $77: 30$ & $1610 \pm 120$ & & & A.D. 340 \\
\hline-20 & $77: 30$ & $1380 \pm 120$ & & & A.D. 570 \\
\hline-21 & $77: 30$ & $1650 \pm 120$ & & & A.D. 300 \\
\hline-22 & $77: 30$ & $1200 \pm 120$ & & & A.D. 750 \\
\hline-23 & $77: 30$ & $3290 \pm 140$ & & & 1340 B.C. \\
\hline-24 & $77: 30$ & $3070 \pm 140$ & & & 1120 B.C. \\
\hline-25 & $77: 29$ & $2030 \pm 130$ & & & 80 B.C. \\
\hline-26 & $77: 29$ & $1910 \pm 130$ & & & A.D. 40 \\
\hline-27 & $77: 31$ & $550 \pm 120$ & & & A.D. 1400 \\
\hline
\end{tabular}

